ISSN 1997-5902

\title{
L'huile des graines de neem, un fongicide alternatif à l'oxyde de cuivre dans la lutte contre la pourriture brune des cabosses de cacaoyer en Côte d'Ivoire
}

\author{
POHE Jean, AGNEROH Thérèse Atcham* \\ Institut National Polytechnique Félix Houphouët Boigny, DFR Agriculture et Ressources Animales, Laboratoire de \\ Phytopathologie et de Biologie Végétale, BP 1313 Yamoussoukro, Côte d'Ivoire \\ Auteur correspondant : tatchamagneroh@yahoo.fr \\ Original submitted in on $16^{\text {th }}$ November 2012. Published online at www.m.elewa.org on $27^{\text {th }}$ February 2013.
}

\section{RESUME}

Objectif : L'effet fongicide de l'huile extraite des graines de neem a été étudié au champ et comparé à celui d'un produit homologué, le Ridomil 66 WP, à base de cuivre sur la pourriture brune des cabosses de cacaoyer due à Phytophthora spp. en Côte d'Ivoire.

Méthodologie et résultats: Trois doses d'huile de neem 10, 15 et $20 \mathrm{~L} / \mathrm{ha}$, appliquées chaque semaine sous forme de bouillies réalisées avec $200 \mathrm{~L}$ d'eau, ont été testées dans un dispositif en blocs complètement randomisés et comparées à un fongicide homologué composé de $60 \%$ d'oxyde de cuivre et $6 \%$ de métalaxyl et pulvérisé toutes les trois semaines à $132 \mathrm{~g} / \mathrm{ha}$. Les résultats portant sur les niveaux de la maladie ont montré que l'huile de neem est statistiquement autant efficace $(P<5 \%)$ à partir de sa petite dose que le produit de référence à la dose homologuée. Elle agit en moins d'une semaine et sa persistance s'étale sur trois semaines.

Conclusion: L'huile des graines de neem a un effet positif dans la lutte contre la pourriture brune des cabosses de cacaoyer. Son efficacité croît avec l'augmentation des doses entre 10 et $15 \mathrm{~L} /$ ha avant de se stabiliser, si bien qu'à partir de la dernière une augmentation de dose n'apporte aucun effet supplémentaire.

Application actuelle et potentiel des résultats : L'huile extraite des graines de neem a un effet fongicide sur la pourriture brune des cabosses de cacaoyer et pourrait être utilisé dans le cadre d'une méthode de lutte respectueuse de l'environnement.

Mots clés : Neem, efficacité, effet fongicide, pourriture brune du cacaoyer, Côte d'Ivoire.

Neem seed oil, an alternative fungicide to copper oxide in the control of brown rot of cocoa pods in Cote d'Ivoire.

Objective: The fungicidal effect of oil extracted from neem seeds has been studied in field conditions and compared with Ridomil 66 WP, an approved copper based product on brown rot of cocoa pods caused by Phytophthora spp. in Cote d'Ivoire.

Methodology and Results: Three doses of neem seed oil 10, 15 and $20 \mathrm{~L} /$ ha applied every week in the form of mixtures done with $200 \mathrm{~L}$ of water, were tested in a randomized complete design and compared with a 
fungicide composed of $60 \%$ copper oxide and $6 \%$ of metalaxyl and sprayed once every three weeks at 132 $\mathrm{g} / \mathrm{ha}$. The results on the levels of the disease showed that neem oil is statistically $(P<5 \%)$ as effective from $10 \mathrm{~L} / \mathrm{h}$ a that the reference product in the approved dose. The neem mixture reacts in less than a week and its persistence is spread over three weeks.

Conclusion: Neem seed oil has a positive effect in the control of brown rot of cocoa pods. Its efficacy increases with increasing doses between 10 and $15 \mathrm{~L} /$ ha before being stabilized, so that from the last dose escalation provides no additional effect.

Current application and potential outcomes: Oil extracted from neem seeds has a fungicidal effect on brown rot of cocoa pods and could be used as part of a method of control which is respectful of environment.

Key words: Neem, efficacy, fungicidal effect, brown rot of cocoa, Cote d'Ivoire.

\section{INTRODUCTION}

Le neem (Azadirachta indica A. Juss.) appelé aussi margousier ou lilas des Indes est un arbre originaire de l'Inde qui fait partie de la famille des Méliacées. II pousse dans les pays subtropicaux de l'Asie et de l'Afrique. II a été implanté en Australie, en Amérique du Sud et Centrale et aux Antilles. Sa feuille composée comprend 9 à 15 folioles de couleur vert foncé. Ses fruits en grappes, qui vont du jaune verdâtre au vert, sont lisses et en forme d'olive. Ils mesurent environ 2 $\mathrm{cm}$ de long et contiennent une pulpe entourant une graine ou amande. Le margousier peut atteindre 25-30 m de haut. II a retenu l'attention des chercheurs à cause de toutes les parties de la plante qui peuvent être utilisées dans la lutte phytosanitaire. Cependant, ce sont les graines qui comportent plus de substances actives dont l'Azadirachtine. A maturité un arbre de neem peut produire $350 \mathrm{~kg}$ de feuilles et $50 \mathrm{~kg}$ de fruits qui fournissent $30 \mathrm{~kg}$ d'amandes. De ces dernières on peut extraire par un procédé de pressage artificiel ou à froid de l'huile (Bélanger et Musabyimana, 2005, Wang et al., 2010).

Avec une production annuelle de 1,2 million de tonnes, la Côte d'Ivoire est le premier producteur mondial de cacao (Centre National de Recherche Agronomique, 2005). La culture est pratiquée dans les grandes régions forestières, notamment au Centre Est et au Sud Ouest du pays. Le système de culture est resté extensif, ce qui a contribué dans une large mesure à la dégradation du couvert forestier. En conséquence, le cacaoyer reste encore à la merci de nombreuses maladies et des ravageurs. Parmi les maladies fongiques, la pourriture brune constitue actuellement la principale source de perte de productivité dans la cacaoyère ivoirienne. Elle est en recrudescence ces dernières années en Côte d'Ivoire (Kouamé, 2006), principalement dans la zone frontalière avec le Ghana, entraînant des pertes de $25 \%$ à 75 $\%$ voire la totalité de la production peut-être à cause de l'apparition récente de l'espèce agressive qu'est Phytophthora megakarya (Koné, 1999).

a lutte se fait par l'utilisation de produits chimiques en général à base de cuivre (Direction de la Protection des Végétaux, 2011 ; Pohé, 2012). Cependant elle pose de nombreux problèmes dont :

a résistance des champignons à certains produits,

-

es effets néfastes des produits sur des insectes utiles et sur l'environnement,

ar ailleurs la cherté de ces produits limite les jeunes producteurs aux moyens financiers modestes.

Dans la recherche des méthodes alternatives de lutte respectueuses de l'environnement (Glitho et al., 2008), il nous a paru opportun d'orienter nos 
travaux sur les extraits végétaux dont celui de neem. L'extrait de neem est connu pour ses propriétés insecticides (Karnavar, 1987 ; Saxena, 1990 ; Schmutterer, 1990; Helson, 1992; Aggarwal et Brar, 2006 ; Mamadou Faye, 2010 ; Gnago et al., 2011). II est cité aussi comme une substance ayant une action fongicide (Häseli et Weibel, 2004 ; Bélanger et Musabyimana, 2005 ; Wang et al., 2010). Ces deux caractéristiques seraient susceptibles d'ouvrir une voie nouvelle dans la lutte dans nos cultures qui sont généralement soumises à un complexe parasitaire. Mais si dans le premier cas, les travaux sont nombreux et la fiabilité des résultats a conduit les pays comme les États Unis et le Canada à homologuer l'Azadirachtine, matière active du neem comme insecticide (Anonyme, 2000;

\section{MATERIEL ET METHODES}

Site expérimental: L'essai a été installé dans une plantation villageoise à Djémissikro, village frontalier avec le Ghana situé à une trentaine de kilomètres de la ville d'Aboisso sur l'axe Aboisso-Noé, au Sud-Est de la Côte d'Ivoire. La zone est caractérisée par un climat de type forestier avec quatre saisons (deux saisons pluvieuses intercalées de deux sèches).

Matériels: Matériel végétal: Les essais ont été réalisés sur des cacaoyers issus d'hybridations diverses dans une plantation villageoise de 12 ans. Produit naturel et fongicide testés : Le produit naturel à expérimenter est l'extrait d'huile des graines de neem. II s'agit d'une solution à base d'amandes de neem directement pressées. II se présente sous forme de concentré émulsionnable (EC). Le produit de référence est le Ridomil 66 WP, un composé de matières actives constituées de $60 \%$ d'oxyde de cuivre et de $6 \%$ de métalaxyl. II est sous forme de poudre mouillable. II est déjà vulgarisé sur le cacaoyer en Côte d'Ivoire. Le produit naturel et le fongicide sont appliqués sous forme de bouillies en mélange avec de l'eau (200 L).

Matériels de traitement: Toutes les applications ont été réalisées à l'aide d'un atomiseur à dos de 16 litres à débit réglable.

Méthodes: Dispositif expérimental: Le dispositif en blocs complètement randomisés est utilisé pour l'étude. II est constitué de cinq traitements sur trois répétitions :
Bélanger et Musabyimana 2005), dans le dernier cas peu de données sont disponibles. C'est dans ce cadre que nous y avons contribué en orientant nos recherches sur les propriétés fongicides d'un biopesticide d'huile des graines de neem avec un test d'efficacité sur la pourriture brune des cabosses de cacaoyer due à Phytophthora spp. L'objectif de cette étude est d'évaluer les taux des cabosses atteintes de pourriture brune dans les parcelles ayant reçu des applications d'huile de graines de neem en comparaison avec ceux obtenus dans les parcelles où les arbres sont traités avec un fongicide composé d'oxyde de cuivre et de métalaxyl déjà homologué en Côte d'lvoire d'une part et d'autre part avec ceux enregistrés avec des arbres sans application fongicide.

- T0 : témoin sans application fongicide ;

- T1: Traitement au complexe fongicide $60 \%$ d'oxyde de cuivre et $6 \%$ de métalaxyl à raison de 132 $\mathrm{g} / \mathrm{ha}$ toutes les 3 semaines selon les recommandations du Ministère de l'Agriculture;

- T2: Traitement à l'huile de graines de neem, à la dose de $10 \mathrm{~L} / \mathrm{ha}$ chaque semaine ;

- T3: Traitement à l'huile de graines de neem, à la dose de $15 \mathrm{~L} /$ ha chaque semaine;

- T4: Traitement à l'huile de graines de neem, à la dose de $20 \mathrm{~L} / \mathrm{ha}$ chaque semaine.

Les répétitions constituent des unités expérimentales ou parcelles élémentaires de $625 \mathrm{~m}^{2} \quad(0,0625 \mathrm{ha})$ chacune, pour un total de $9375 \mathrm{~m}^{2}$ (0,9375 ha). Les parcelles élémentaires sont au nombre de 3 par traitement soit 15 parcelles élémentaires au total. Le nombre de plants par parcelle élémentaire est de 83 pieds soit 1245 cacaoyers pour l'ensemble des parcelles élémentaires. Par parcelle élémentaire, dix pieds sont mis en observation soit 150 cacaoyers observés pour tout le test. La mise en place de l'essai et le premier traitement ont eu lieu le 22 Juillet 2011, période caractérisée par une pluviométrie favorable au développement de la pourriture brune des cabosses de cacaoyer (Brasier et Hansen, 1992). Les applications fongicides: Les bouillies sont appliquées les après midis et chaque semaine après observation du niveau 
de la maladie dans toutes les parcelles élémentaires. Elles ont été arrêtées après trois semaines et seules les observations portant sur le niveau de la maladie ont été poursuivies.

Paramètres observes: Les observations hebdomadaires ont porté sur:

- Le nombre de cabosses nouvellement atteintes sur la base des symptômes décrits par Babacauh (1975);

- Le nombre total de cabosses.

\section{RESULTATS}

Le temps de réponse : II s'agit ici de montrer à partir de quelle période de son application, le produit a un effet significatif sur le niveau de la maladie. Les analyses portant sur les taux d'attaques sont faites à partir des observations du premier jour. L'analyse de variance appliquée sur les données enregistrées a
Les récoltes sanitaires sont pratiquées à chaque passage hebdomadaire sur toutes les parcelles élémentaires.

Analyse des données: Les taux des cabosses pourries ont été calculés par rapport au nombre total de cabosses observées. Les données relatives à l'impact des applications fongicides sur une ou trois semaines ont été soumises à une analyse de variance portant sur ces taux et la comparaison des moyennes de ceux-ci par la plus petite différence significative (PPDS) au seuil de probabilité $p<5 \%$.

montré que les niveaux d'attaques de Phytophthora sont statistiquement identiques dans toutes les parcelles à la première observation, c'est-à-dire avant la pulvérisation des produits fongicides sur les cacaoyers (Tableaux 1 et 2).

Tableau 1 : Taux (en \%) des cabosses de cacaoyer attaquées par Phytophthora spp. avant l'application fongicide.

\begin{tabular}{llllc}
\hline Traitements & Rép1 1\%) & \multicolumn{1}{c}{ Rép2 (\%) } & \multicolumn{1}{c}{ Rép3(\%) } & Moyenne (\%) \\
\hline T0 & 36,00 & 28,79 & 30,70 & 31,0 \\
T1 & 33,33 & 25,95 & 29,46 & 29,58 \\
T2 & 24,48 & 38,39 & 30,40 & 30,53 \\
T3 & 34,19 & 29,17 & 2,60 & 30,95 \\
T4 & 32,84 & 40,57 & 34,17 & 35,56 \\
\hline
\end{tabular}

$\mathrm{T} 0=$ Témoin non traité; $\mathrm{T} 1=$ Produit de référence; $\mathrm{T} 2=$ Huile de neem $10 \mathrm{~L} / \mathrm{ha} ; \mathrm{T} 3=$ Huile de neem

$15 \mathrm{~L} / \mathrm{ha} ; \mathrm{T} 4$ = Huile de neem $20 \mathrm{~L} / \mathrm{ha}$; Rép = répétition.

Tableau 2: Analyse statistique sur les taux des cabosses de cacaoyer attaquées par Phytophthora spp. avant l'application fongicide

\begin{tabular}{|l|l|l|l|l|l|l|}
\hline Sources de variation & DDL & Somme des carrés & Carré moyen & F calculé & \multicolumn{2}{|c|}{ F lu } \\
\cline { 5 - 7 } & & & & & $5 \%$ & $1 \%$ \\
\hline Répétitions & 2 & 7,18 & 3,59 & $\mathbf{0 , 1 5} \mathbf{~ n s}$ & 4,46 & 8,65 \\
\hline Traitements & 4 & 67,00 & 16,75 & $\mathbf{0 , 6 9} \mathbf{~ n s}$ & 3,84 & 7,01 \\
\hline Erreur expérimentale & 8 & 194,08 & 24,26 & & & \\
\hline Total & 14 & 268,26 & & & & \\
\hline
\end{tabular}

Ns : non significatif

Cependant, une semaine après celle-ci, apparait une différence significative entre les traitements. L'analyse de variance portant sur ces taux a révélé une différence significative entre les témoins sans application fongicide et les parcelles traitées aux fongicides (Tableaux 3 et 4 ). 


\section{Pohe et Agneroh.... J. Appl. Biosci. 2013. L'huile des grains de neem, un fongicide alternative de la}

cacaoyer

Tableau 3: Taux des cabosses de cacaoyer attaquées par Phytophthora spp. une semaine après l'application fongicide.

\begin{tabular}{lllcc}
\hline Traitements & Rép1(\%) & Rép2(\%) & Rép3(\%) & Moyenne (\%) \\
\hline T0 & 34,72 & 17,65 & 23,2 & $\mathbf{2 4 , 3 3}$ \\
T1 & 10 & 7,14 & 7,06 & $\mathbf{7 , 9 1}$ \\
T2 & 6,90 & 5,56 & 7,22 & 6,67 \\
T3 & 6,17 & 1,94 & 3,23 & 3,61 \\
T4 & 2,94 & 1,89 & 3,81 & $\mathbf{2 , 8 8}$ \\
\hline
\end{tabular}

T0 = Témoin non traité; $\mathrm{T} 1$ = Produit de référence; $\mathrm{T} 2$ = Huile de neem $10 \mathrm{~L} / \mathrm{ha} ; \mathrm{T} 3$ = Huile de neem $15 \mathrm{~L} / \mathrm{ha}$; T4 = Huile de neem $20 \mathrm{~L} / \mathrm{ha}$; Rép = répétition

Tableau 4: Analyse statistique sur les taux (en \%) des cabosses de cacaoyer attaquées par Phytophthora spp. une semaine après l'application fongicide.

\begin{tabular}{|l|l|l|l|l|l|l|}
\hline \multicolumn{1}{|c|}{ Sources de variation } & DDL & $\begin{array}{l}\text { Somme des } \\
\text { carrés }\end{array}$ & Carré moyen & F calculé & \multicolumn{2}{|c|}{ F lu } \\
\cline { 6 - 8 } & & & & & $5 \%$ & $1 \%$ \\
\hline Répétitions & 2 & 71,59 & 35,80 & $\mathbf{2 , 9 2} \mathbf{~ n s}$ & 4,46 & 8,65 \\
\hline Traitements & 4 & 1001,53 & 250,38 & $\mathbf{2 0 , 3 9}$ ** & 3,84 & 7,01 \\
\hline Erreur expérimentale & 8 & 98,21 & 12,28 & & & \\
\hline Total & 14 & 1171,34 & & & & \\
\hline
\end{tabular}

*: significatif ; ns : non significatif, PPDS $=4,42$ à $5 \%$

$\begin{array}{lllll}\text { Classement des moyennes : } & \text { T0 } & >\mathrm{T} 1 & = & \mathrm{T} 2 \\ 24,23 & 7,91 & 6,67\end{array}=\underset{3,61}{\mathrm{~T} 3}=\underset{2,88}{\mathrm{~T} 4}$

L'analyse par la PPDS a montré une similarité entre les traitements fongicides. Les deux produits comparés avaient tous deux un temps de réponse de moins d'une semaine.

Le niveau d'efficacité : L'effet de l'huile des graines de neem sur la pourriture brune a été évalué en comparant les taux des attaques dans les parcelles soumises à son traitement avec ceux du traitement du produit de référence d'une part et d'autre part avec ceux obtenus dans les témoins sans application fongicide. Ces différents taux d'attaques obtenus sont représentés dans la Figure 1.

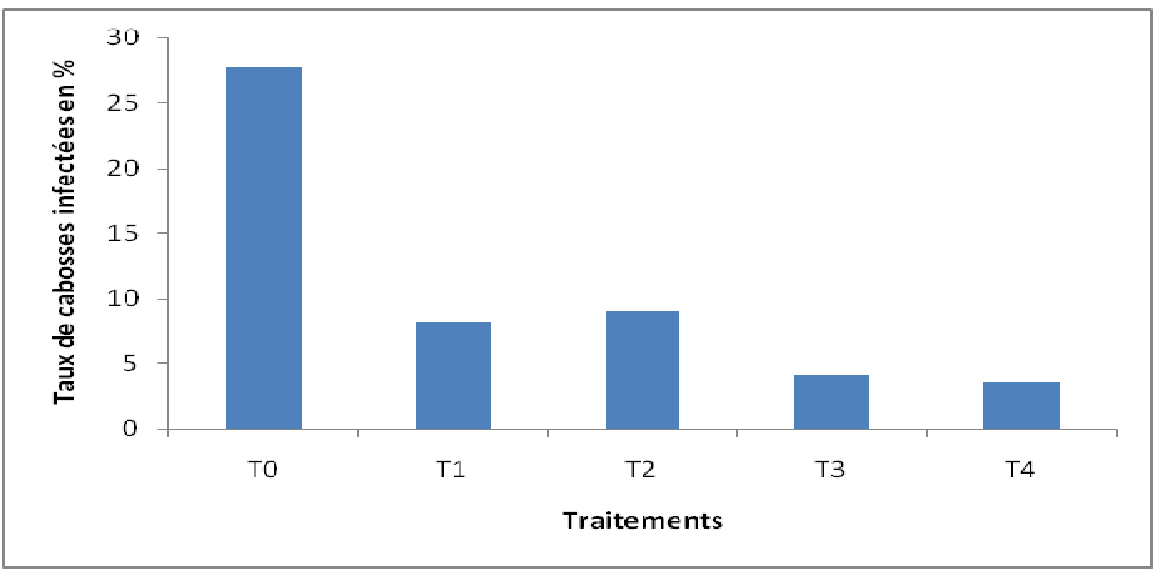

Figure 1 : Taux moyens (en \%) des attaques de Phytophthora spp. dans différentes parcelles expérimentales après trois semaines d'application fongicide. 
L'analyse de la Figure 1 laisse apparaître de fortes différences entre les taux obtenus dans les parcelles témoins et ceux des parcelles ayant reçu des applications fongicides. En effet, dans les parcelles sans application fongicide (T0), les niveaux des attaques restent très élevés soit environ $27,73 \%$, alors que ceux des parcelles traitées aux fongicides sont devenus bas avec une égalité apparente entre les parcelles traitées à l'huile de neem à la dose de 10 $\mathrm{L} / \mathrm{ha}$ (T2) et celles traitées au produit de référence
$(9,04 \%$ et $8,18 \%)$. Aussi l'augmentation de la dose d'huile de neem de 15 à 20 L/ha n'a-t-elle pas apporté d'effet complémentaire significatif $\left(4,14 \%\right.$ et $\left.3,52 \%{ }^{\circ}\right)$. Ces deux dernières doses, d'effet apparemment identique, se montrent plus efficaces que le produit de référence à sa dose homologuée. Les analyses statistiques par l'analyse de variance (Tableau 5) et la comparaison des moyennes appliquées à ces taux confirment ces observations.

Tableau 5: Analyse statistique sur les taux (en \%) des cabosses de cacaoyer attaquées par Phytophthora spp. après trois semaines d'application fongicide.

\begin{tabular}{|c|c|c|c|c|c|c|}
\hline \multirow[t]{2}{*}{ Sources de variation } & \multirow[t]{2}{*}{ DDL } & \multirow{2}{*}{$\begin{array}{l}\text { Somme des } \\
\text { carrés }\end{array}$} & \multirow{2}{*}{$\begin{array}{l}\text { Carré } \\
\text { moyen }\end{array}$} & \multirow[t]{2}{*}{ F calculé } & \multicolumn{2}{|c|}{ Flu } \\
\hline & & & & & $5 \%$ & $1 \%$ \\
\hline Répétitions & 2 & 55,49 & 27,74 & $3,58 \mathrm{~ns}$ & 4,46 & 8,65 \\
\hline Traitements & 4 & 1235,00 & 308,75 & $39,89^{* *}$ & 3,84 & 7,01 \\
\hline Erreur expérimentale & 8 & 61,91 & 7,74 & & & \\
\hline Total & 14 & 1352,40 & & & & \\
\hline
\end{tabular}

*: significatif ; ns : non significatif

PPDS $=3,51$ à $5 \%$

Classement des moyennes: $\mathrm{T} 0>\mathrm{T} 2=\mathrm{T} 1>\mathrm{T} 3=\mathrm{T} 4$

$27,73 \quad 9,04 \quad 8,18 \quad 4,14 \quad 3,52$

Persistance de l'efficacité: Les taux des attaques sont restés bas sur une période après l'arrêt des applications de l'huile de neem en S2, S0 correspondant à la première application (Figure 2). Sur cette Figure, l'action de l'huile de neem est demeurée effective sur trois semaines (S2 à S5), période à la fin de laquelle le niveau de la maladie a connu une montée régulière.

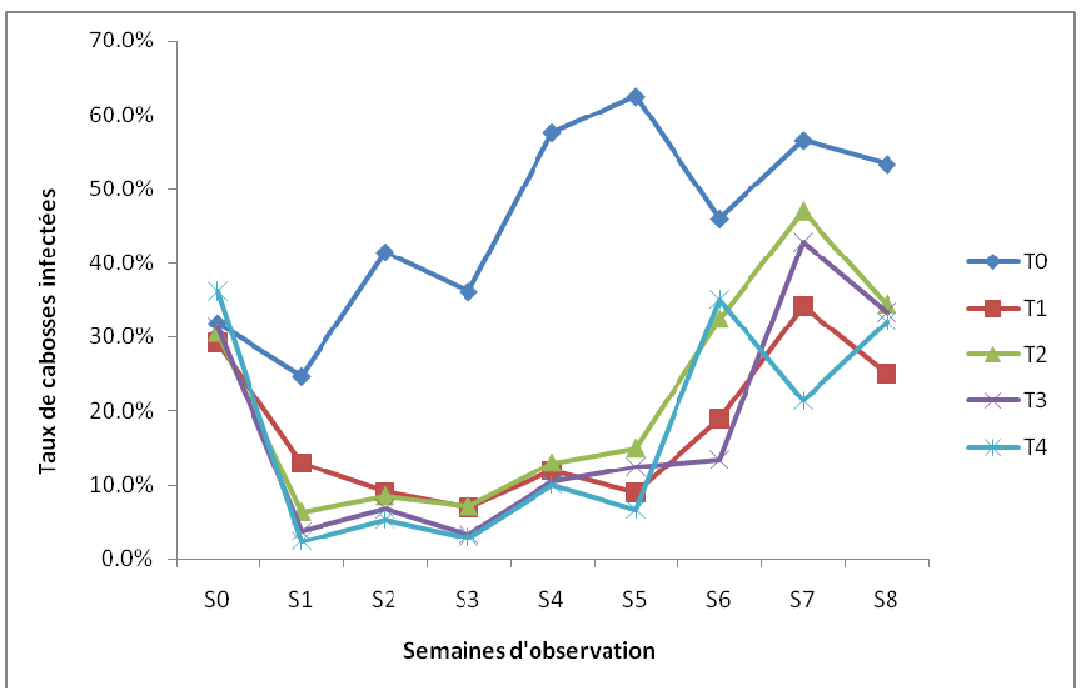

Figure 2: Évolution hebdomadaire des taux de cabosses infectées par Phytophthora spp. 


\section{DISCUSSION}

Les résultats de ces travaux montrent un effet significatif positif de l'extrait d'huile de neem dans la réduction des attaques des Phytophthora spp. sur les cabosses de cacaoyer. Ces résultats apparaissent comme une preuve de plus sur les propriétés fongicides du neem déjà mentionnées par un certain nombre de chercheurs. Häseli et Weibel (2004) ont montré l'efficacité des extraits de neem sur les maladies dans la production des cerises. Bélanger et Musabyimana (2005) les ont mentionnés comme étant efficaces sur un certain nombre de champignons responsables des maladies des plantes cultivées (Microsphaera sp., sur le rosier, Botrytis cinerea sur la fraise, Alternaria panax sur le ginseng). Au canada où il bénéficie d'une homologation temporaire en tant qu'insecticide (Anonyme, 2000), le neem et surtout sa composante l'Azadirachtine a fait l'objet de nombreux tests au laboratoire, en serre et au champ dans le domaine de l'horticulture et de la foresterie avec des résultats positifs aussi bien sur les insectes que sur les champignons (Roger, 1992; Bélanger et Musabyimana, 2005). Au Brésil, tous les producteurs et distributeurs de produits agricoles connaissent les extraits de neem dont les vertus sont multiples et dont ils en font une utilisation courante pour protéger les fruits et légumes (Silva et al., 2011 ; de Oliveira et al., 2012).. Les effets des extraits de neem sur les champignons pathogènes comme Phytophthora inféodées à la pomme de terre et à la tomate et Oidium $\mathrm{du}$ haricot $\mathrm{y}$ ont été rapportés (Bélanger et Musabyimana, 2005). Ils sont considérés par des chercheurs comme des substances à plusieurs vertus. Ils servent à lutter aussi bien contre les maladies des

\section{CONCLUSION}

Cette expérimentation effectuée en plein champ vient une fois de plus confirmer l'efficacité du fongicide de référence, déjà homologué dans la lutte contre la pourriture brune des cabosses de cacaoyer en Côte d' Ivoire. Aussi les résultats obtenus ont-ils permis de mettre en évidence l'efficacité de l'extrait d'huile des graines de neem. Cette substance appliquée à la dose de $10 \mathrm{~L} /$ ha est statistiquement autant efficace que le produit de référence à sa dose homologuée. Elle est capable de réduire considérablement l'incidence de la hommes que celles des plantes (Kausik et al., 2002). En plus de leurs actions insecticides et fongicides, elles seraient antivirales (Rao et al., 1989) et bactéricides (Shamapada et al., 2007) Parmi les propriétés des extraits de neem par lesquelles ils agissent sur les insectes à savoir les effets répulsif, anti appétant et régulateur de la croissance (Gill et Lewis, 1971; Karnavar, 1987 ; Osman et Port, 1990 ; Weathersbee et Tang; 2002; Isman, 2006 ; Gueye et al., 2011), seule leur action sur la croissance et le développement des champignons a été rapportée (Kabeh et Jalingo, 2007). Les extraits des feuilles de neem et surtout des graines plus riches en Azadirachtine, capables de contrôler à la fois les ravageurs et les maladies des plantes ouvrent une voie de lutte intégrée. Ils constitueraient même une alternative comme le propose un certain nombre de chercheurs, car contrairement aux pesticides de synthèse, leur utilisation présenterait une innocuité pour l'environnement, et serait sans effet sur les faunes bénéfiques et anotoxique pour l'homme (Roger, 1992; Schenk et al., 2001 ; Bélanger et Musabyimana, 2005 ; Condor-Golec, 2007 ; Regnault-Roger, 2007). Ils sont appliqués à faible quantité. Dans le cas précis de nos observations, nous obtenons une réponse satisfaisante à partir de $10 \mathrm{~L} / \mathrm{ha}$ et avec des applications qui peuvent être espacées de trois semaines, ce qui réduirait les coûts de traitement. Pour toutes ces raisons nous considérons l'extrait d'huile des graines de neem, comme un fongicide espoir pour nos producteurs de cacao qui sans doute amélioreront leur productivité.

pourriture brune des cabosses de cacaoyer. Agissant sur cette maladie due aux champignons, son effet fongicide ne saurait constituer un doute et elle peut jouer un rôle important dans la lutte intégrée dans nos cultures et même constituer une alternative aux pesticides de synthèse dont l'utilisation n'est pas sans effet sur l'homme et son environnement. Ces extraits peuvent être fabriqués par les paysans eux-mêmes s'ils disposent de broyeurs ou moulins et de presses adaptées pour leur extraction. 


\section{REFERENCES BIBLIOGRAPHIQUES}

Anonyme, 2000. Nouvelles et conseils de l'Agence de réglementation de la lutte antiparasitaire au Canada. INFO ARLA no 2, 2 Nov. 2000.

Aggarwal N and Brar D S, 2006. Effects of different neem preparations in comparison to synthetic insecticides on the whitefly parasitoid Encarsia sophia (Hymenoptera: Aphelinidae) and the predator Chrysoperla carnea (Neuroptera: Chrysopidae) on cotton under laboratory conditions. J Pest Sci 79: 201-207.

Babacauh KD, 1975. Évolution de la pourriture brune dans les conditions naturelles de la cacaoyère ivoirienne. Café Cacao Thé 19 : 293-301.

Bélanger A et Musabyiamana T, 2005. Le neem contre les insectes et les maladies. Journées Horticoles au Canada en 2005. 4 pages.

Brasier CM and Hansen EM, 1992. Evolutionary biology of Phytophthora, Part II: Phylogeny, speciation and population structure. Annu. Rev. Phytopathol. 30: 173-200.

Centre National de Recherche Agronomique, 2005. Bien cultiver le cacaoyer en Côte d'Ivoire. 4 pages.

Condor-Golec AF, 2007. Effect of neem (Azadirachta indica A. Juss) insecticides on parasitoids. Rev. per. biol. 14(1): 69-74.

De Oliveira $F Q$, Malaquias JB, de Souza Figueiredo WR, de Luna Batista J, Barbosa Beserra E, 2012. Inhibition of fruit infestation by Mediterranean fruit fly using natural products. African Journal of Biotechnology 11(74): 13922-13927.

Direction de la Protection des Végétaux, 2011. Catalogue phytosanitaire de la Côte d'Ivoire. Ministère de l'Agriculture. Décembre 2011. 98 $p$.

Gill JS and Lewis CT, 1971. Systemic action of an insect feeding deterrent. Nature 232: 402-403.

Glitho IA, Ketoh GK, Nuto PY, Amevoin SK, Huignard $J$, 2008. Approches non toxiques et non polluantes pour le contrôle des populations d'insectes nuisibles en Afrique du Centre et de I'Ouest. In Biopesticides d'origine végétale (2è édition), Regnault $R$, Philogène BJR, Vincent C (eds). Editeur Tec et Doc/ Lavoisier : 207-217.
Gnago AJ, Danho M, Agneroh, TA, Fofana, IK, Kohou AG, 2010. Efficacité des extraits de neem (Azadirachta indica) et de papayer (Carica papaya) dans la lutte contre les insectes ravageurs du gombo (Abelmochus esculentus) et du chou (Brassica oleracea) en Côte d'Ivoire. International Journal of Biological and Chemical Sciences 4(4): 953-965.

Gueye MT, Seck D, Wathelet JP, Lognay G, 2011. Lutte contre les ravageurs des stocks de céréales et de légumineuses au Sénégal et en Afrique Occidentale: Synthèse biographique. Biotechnol. Agron. Soc. Environ. 15(1): 183194.

Häseli, A and Weibel F, 2004. Disease control in organic cherry production with new products and early plastic cover of the trees. In Boos Markus (Ed.) Ecofruit $-11^{\text {th }}$ international conference on cultivation technique and problems in organic fruit- growing. $3^{\text {rd }}$ February. - $5^{\text {th }}$ February 2004, Weinsbererg/ Germany. pp 122-130.

Helson B, 1992. Naturally derived insecticides: Prospects for forestry use. Forestry chronicle 68: 349-354.

Isman MB, 2006. Botanical insecticides, deterrents and repellents in modern agriculture and an increasingly regulated world. Ann. Rev. Entomol. 51: 45-66.

Kabeh JD and Jalingo MGDSS, 2007. Mini review exploiting neem (Azadirachta indica). Resources for improving the quality of life in Taraba state, Nigeria. Int. J. Agri-Biol. 9 (3): 530-532.

Karnavar GK, 1987. Influence of azadirachtin on insect nutrition and reproduction. Proceedings of the Indian Academy of Science (Animal Science) 96: 341-347.

Kausik Biswas, Ishita Chattopadhyay, Ranajit K.Banerjee, Uday Bandyopadhyay, 2002. Biological activities and medicinal properties of neem (Azadirachta indica). Current science 82(11): 1336-1345.

Koné YR, 1999. Étude de la structure actuelle des populations de Phytophthora spp. agent de la pourriture brune des cabosses du cacaoyer 
(Theobroma cacao L.) en Côte d'Ivoire. Mémoire de fin d'études en vue de l'obtention du Diplôme d'Agronomie Approfondie, Option défense des cultures. École Supérieure d'Agronomie. Yamoussoukro, 111 pages.

Kouamé KD, 2006. Structure et dynamique des populations de Phytophthora spp., agents de la pourriture brune des cabosses du cacaoyer (Theobroma cacao L.) en Côte d'Ivoire. Mémoire de DEA, UFR Biosciences. Université de Cocody, Abidjan, Côte d'Ivoire. 74 pages.

Mamadou Faye, 2010. Nouveau fractionnement de la graine de neem (Azadirachta indica Juss) sénégalais: production d'un biopesticide d'huile et de tourteau. Thèse en vue de l'obtention du Doctorat de I'Université de Toulouse délivré par l'Institut Polytechnique de Toulouse (INP Toulouse). 267 pages.

Osman M Z and Port GR, 1990. Systemic action of neem seed substances against Pieris brassicae. Entomologia Experimentalis et Applicata 54: 297-300.

Pohé J, 2012. Action du dosage en cuivre des sels cupriques et de la périodicité de leur application sur la pourriture brune des cabosses de cacaoyer en Côte d'lvoire. Journal of Animal and Plant Sciences 15(3): 2243-2251.

Rao AR, Sukumar SS, Paramasivarm TB, Kamalakshi S, Parashurama AR, Shanta M, 1969. Study of antiviral activity of tender leaves of Margosa tree (Melia azadericta) on vaccinia and variola virus: a preliminary report. Ind. J. Med.Res. 57(3): 495-502.

Regnault-Roger,C, 2007. Biopesticides : une alternative aux pesticides conventionnels ? Mer, Littoral, Lacs et Cours d'eau 73: 60-64.

Roger, C. 1992. Impact des pesticides chimiques et biologiques sur la survie et l'efficacité de prédation de Coleomegilla maculata lengi Timberlake (Coleoptera: Coccinellidae). Mémoire de maitrise en Biologie. Université du Québec à Montréal, Québec-Canada, 76 pages.

Saxena R.C. 1993. Neem as a source of natural insecticides-an update. In Proceed. Symp. Botanical Pesticides in IPM. Indian Soc. Tobacco science, pp 1-24, Rajahmundry, India.

Silva MA, Alvarenga CD, Bezerra- Silva GCD, Mastrangelo T, Lopes-Mielezaski GN, Giustolin T, 2011. Toxic effects of neem seed cake on the larval-pupal (prepupal) stage of Mediterranean fruit fly (Diptera: Tephritidae). Fruits 66(5): 363-369.

Schenk P, Imdorf A et Fluri P, 2001. Effets de l'huile de neem sur l'acarien varroa et les abeilles. Revue suisse d'apiculture 98(3): 114-119.

Schmutterer $H, 1990$. Properties and potential of natural pesticides from the neem tree, Azadirachta indica. Ann.Rev. Entomol. 35: 271-297.

Shyamapada Mandal, Manisha Deb Mandal, Nishith Kumar Pal, 2007. Antibacterial potential of Azadirachta indica seed and Bacopa monniera leaf extracts against multidrug resistant Salmonella enteric serovar Typhi isolates. Arch Med Sci 31: 14-18.

Wang Jingfa, Li Jian, Cao Jiankang, Jiang Weibo, 2010. Antifungal activities of neem (Azadirachta indica) seed kernel extracts on post harvest diseases in fruits. African Journal of Microbiology Research 4(11): 1100-1104.

Weathersbee AA and Tang YQ, 2002. Effect of neem seed extract on feeding, growth, survival and reproduction of Diaprepes abbreviates (Coleoptera: Curculionidae). Journal of Economic Entomology 95(4): 661-667. 